\title{
Micromechanical Modelling of Superelasticity in Shape Memory Alloys
}

\author{
E. Patoor, A. Eberhardt and M. Berveiller \\ Laboratoire de Physique et Mécanique des Matériaux, URA 1215 du CNRS, Institut Supérieur de Génie \\ Mécanique et Productique, Université de Metz, Ile du Saulcy, 57045 Metz cedex, France
}

\begin{abstract}
Micromechanical methods developed to describe the thermomechanical behavior of solids are applied to phase transition related problem. Results obtained are compared with those obtained using a macroscopic phenomenological approach. This micromechanical analysis is based on a kinematical description of the physical strain mechanisms and a definition of a local thermodynamical potential. Volume fractions of the different variants of martensite are chosen as internal variables to describe the evolution of the microstructural state of the material. This analysis determines local constitutive equations for the behavior. Global relationships are obtained using a self consistent scheme. This approach gives results in good agreement with experimental observations performed on $\mathrm{Cu}-$ based Shape Memory alloys.
\end{abstract}

\section{INTRODUCTION}

In recent years much interest was centered on the micromechanical approaches of the thermomechanical behavior of solids. Developments of these approaches to linear (such as thermoelasticity) or non linear problem (such as elasto-plasticity) had established their workability and versatility. These advances are related with defects theory developed from the works of Eshelby and Kröner. Using scale transition method issued from the field theory (integral equation, self-consistent approximation) gives a successful description for thermomechanical properties. Application of these recent developments in mechanics of materials is able to contribute in the description of the phase transition related problems.

Phase transitions occurring in solid state produce large effects on the thermomechanical behavior. In most cases, these problems deal with coupling phenomena between the thermomechanical response associated to phase transitions and other inelastic strain mechanisms, like plasticity by moving dislocations. These coupling make an accurate description of the behavior very difficult to perform. In this context, Shape Memory Behavior is a particular interesting case. In a first approximation, the transformation strain is the only inelastic strain mechanism in these materials.

The first part of this presentation gives a phenomenological description of phenomena observed in shape memory alloys. Strain mechanisms at the origin of these behaviors and microstructural observations are detailed. The second part deals with a kinematical description of the transformation. In that part, microstructural aspects are successively described using a global approach, a crystallographical model and a moving boundaries framework. Results obtained using these different scales are discussed. From this analysis the crystallographical approach seems well-adapted to model the shape memory alloys behaviors . Thermodynamical potential associated to a martensitic phase transition is derived in the third part. This potential is at first defined using a global two phases approach. Major limitations related to this macroscopic description are underlined. These troubles are avoid using scale transition method that is detailed in the fourth part. In this framework, thermodynamical potential is determined for an elementary representative domain. Grain scale is chosen as micro level. To account with the growing of several variants of martensite inside grain an interaction matrix is defined from micromechanical analysis. From these considerations a local constitutive equation is obtained for the single crystal behavior. Volume fractions of the different variants of martensite are used to describe the internal state evolution of the material during the phase change.

Overall response is determined using a self-consistent approximation of the thermomechanical integral equation. The material response is then determined from the definition of a limited set of physical parameters. It is important to notice that all the parameters used in this model can be determined experimentally. 
Application of scale transition approach to stress-induced thermoelastic martensitic transformation is developed in part five. Results such obtained are in good agreement with experimental observations performed on $\mathrm{Cu}$-based Shape Memory alloys. From computed loading surface obtained using this micromacro framework a macroscopic transformation criterion directly applicable to structure calculation is derived in part six .

\section{PHENOMENOLOGICAL AND MICROSTRUCTURAL OBSERVATIONS}

Several thermomechanical behaviors are observed in shape memory alloys. All these phenomena are related to the typical properties of the thermoelastic martensitic transformation :

- Stress-induced martensitic transformation leads to the superelastic behavior [1].

- Self-accommodation in thermally induced martensite associated to a very large mobility of intervariant boundaries in the martensitic state are responsible to the so-called shape memory effect [2].

- Influence of the internal stress field generates by a microstructure of oriented defects gives the spectacular two-way memory [3].

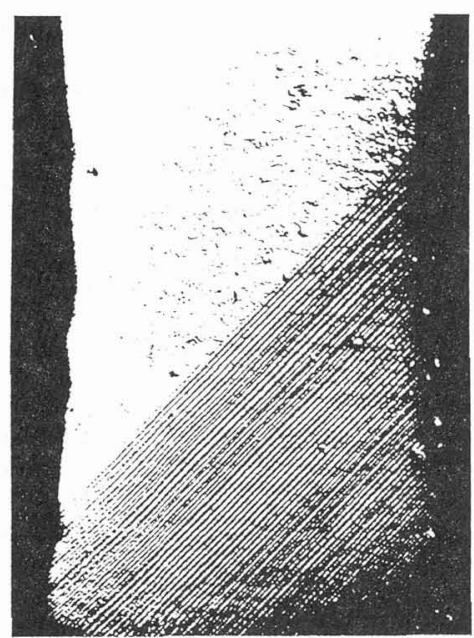

(a)

$$
\stackrel{300 \mu \mathrm{m}}{\longmapsto}
$$

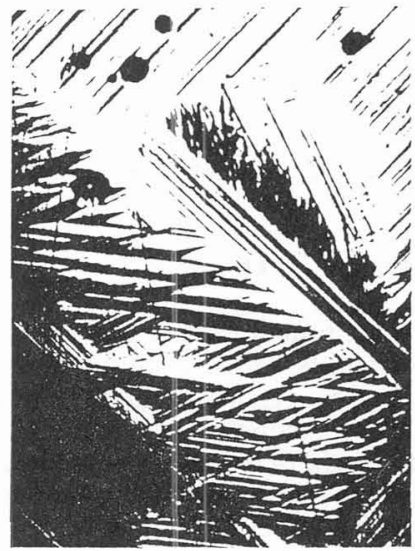

(b)



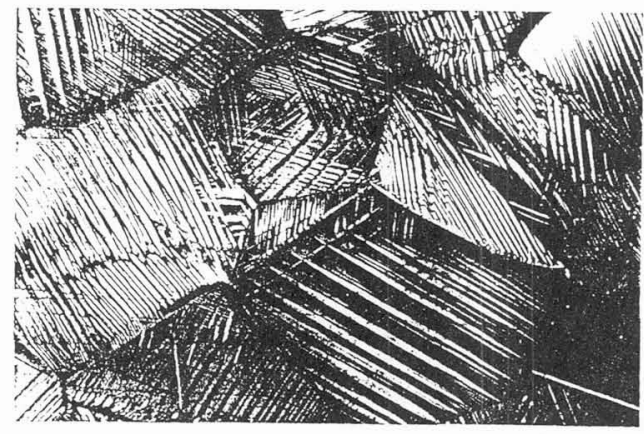

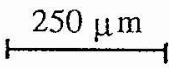

(c)

Figure 1: Micrographies showing different typical morphologies for the martensitic phase in $\mathrm{Cu}-\mathrm{Zn}-\mathrm{Al}$ shape memory alloys :

(a) Stress-oricnted growth in a single crystal during tensile test.

(b) Self accommodation structure induced by a stress-free cooling.

(c) Multivariant transformation inside grain for uniaxial tensile test performed on polycrystalline sample. 
Two elementary strain mechanisms appear in these three particular behaviors. First is a preferentially growth of the martensite plates with respect to the stress field (applied or internal). This is responsible to the occurrence of a large spread of morphology for the martensitic phase, from a fully oriented one in single crystal transformed under uniaxial tensile stress (figure 1.a) to a self-accommodated pattern when transformation occurs without any stress assistance (figure 1.b). Intermediate states are observed inside grains in polycrystalline material submitted to a uniaxial tension test (figure 1.c).

The second strain mechanism related to this solid state transformation is the very high mobility of interfaces between the martensitic domains. This phenomenon is responsible to shape memory and rubberlike effects but it appears too in stress-induced transformation (for non proportional loading paths for instance).

This work deals mainly with stress-induced transformation aspects. It may be noticed that the very flat plateau obtained in that case on single crystal (figure 2) is related to the growth of a unique variant of martensite stress-induced. Such plateau is not observed on polycrystalline sample (figure 3 ). In that case, granular structure is responsible to the formation of several variants of martensite in each grain. Dissymmetry between tensile and compressive test curve originates from these microstructural considerations [4].

In any case, the superelastic behavior is strongly dependent on the temperature (figure 2) and despite the thermoelastic character of the transformation, a hysteretic behavior is obtained on unloading (figure 3).

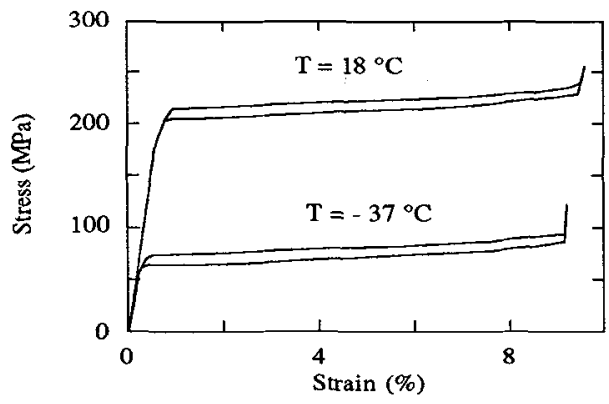

Figure 2: Influence of the temperature on the superelastic behavior observed in the single crystal case. $\mathrm{Cu}-\mathrm{Zn}-\mathrm{Al}\left(\mathrm{M}_{\mathrm{s}}=-60^{\circ} \mathrm{C}\right)[5]$.

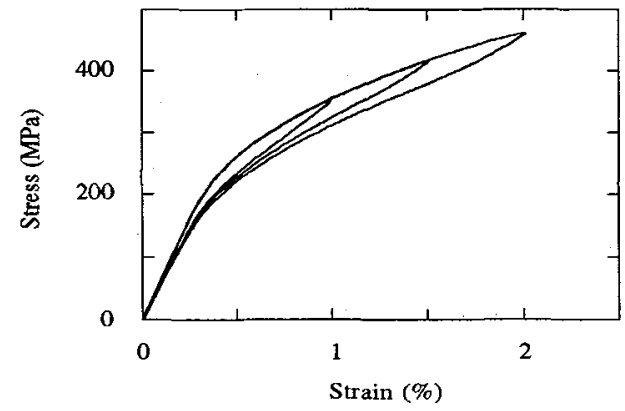

Figure 3: Polycrystalline superelastic stress-strain curve showing sub-loop trajectories.

$\mathrm{Cu}-\mathrm{Al}-\mathrm{Be}\left(\mathrm{M}_{\mathrm{s}}=-100^{\circ} \mathrm{C}\right)$ at $\mathrm{T}=-20,5^{\circ} \mathrm{C}[6]$.

\section{KINEMATICAL DESCRIPTION OF THE MARTENSITIC PHASE TRANSITION}

Let us consider a volume $V$ bounded by a surface $\partial \mathrm{V}$. In a stress-free state this volume is fully austenitic at a temperature $T_{0}$ larger than $A_{f}$. Loading condition applied on $\partial V$ (imposed displacement or given force) associated to a uniform temperature change $\Theta\left(\Theta=T-T_{0}\right)$ generate elastic distortion $\beta^{\mathrm{e}}$, thermal one $\beta^{\text {th }}$ and a distortion field $\beta^{\mathrm{T}}(\mathrm{r})$ due to the martensitic transformation. In the infinitesimal strain framework (that is reasonable because $\beta^{T}<1$ ) total distortion field is given by :

$$
\beta_{\mathrm{ij}}(\mathrm{r})=\beta_{\mathrm{ij}}^{\mathrm{e}}(\mathrm{r})+\beta_{\mathrm{ij}}^{\mathrm{th}}(\mathrm{r})+\beta_{\mathrm{ij}}^{\mathrm{T}}(\mathrm{r})
$$

In this analysis influence of plastic distortion (due to a motion of dislocations) is neglected.

When the displacement field $\mathbf{u}(\mathrm{r})$ induced by the loading system is continuous inside volume $V$, total distortion $\beta(r)$ is equal to the gradient of this displacement. Taking only account the pure strain contribution (i.e., symmetrical part of $\beta(r)$ ) leads to:

$$
E_{i j}=\frac{1}{V} \int_{v}\left[\varepsilon_{i j}^{e}(r)+\varepsilon_{i j}^{\text {th }}(r)+\varepsilon_{i j}^{T}(r)\right] d V
$$


where $\mathrm{E}$ denotes the uniform macroscopic strain imposed on $\partial \mathrm{V}$. Thermoelastic laws relate local strains $\varepsilon^{e}(r)$ and $\varepsilon^{\text {th }}(r)$ to the local stress field $\sigma(r)$ and to the local change in the temperature $\Theta(r)$ using the local elastic stiffness tensor $s(r)$ and the local thermal expansion coefficient $\alpha(r)$ :

$$
\varepsilon_{\mathrm{ij}}^{\mathrm{e}}(r)=s_{\mathrm{ijkl}}(r) \sigma_{\mathrm{kl}}(\mathrm{r}) \quad \text { and } \quad \varepsilon_{\mathrm{ij}}^{\text {th }}(r)=\alpha(r) \delta_{\mathrm{ij}} \Theta(r)
$$

where $\delta_{\mathrm{ij}}$ denotes the Kroneker symbol. Using these expressions transforms relation (2) into :

$$
E_{i j}=\frac{1}{V} \int_{V} s_{i j k I}(r) \sigma_{k I}(r) d V+\frac{1}{V} \int_{v} \alpha(r) \delta_{i j} \Theta(r) d V+\frac{1}{V} \int_{v} \varepsilon_{i j}^{T}(r) d V
$$

When elastic and thermal properties are considered as homogeneous inside the material and if the temperature field is kept uniform the three contributions in equation (4) can be identified to the macroscopic elastic, thermal and transformation strains (respectively $E^{e}, E^{\text {th }}, E^{T}$ ). Considering :

$$
\begin{array}{lll}
\mathrm{s}_{\mathrm{ijk} 1}(\mathrm{r})=\mathrm{S}_{\mathrm{ijkl}} \quad \alpha(\mathrm{r})=\alpha \quad \Theta(\mathrm{r})=\Theta \quad \forall \mathrm{r} \in \mathrm{V}
\end{array}
$$

and denoted by $\Sigma$ the mean value of the local stress field $\sigma(r)$ transform equation (4) into :

$$
E_{i j}=S_{i j k l} \Sigma_{k l}+\alpha \delta_{i j} \Theta+E_{i j}^{T}
$$

In that case, the overall transformation strain is defined by :

$$
E_{i j}^{T}=\frac{1}{V} \int_{v} \varepsilon_{i j}^{T}(r) d V
$$

Three kinds of microstructural descriptions appear well adapted to account for discontinuities in the transformation strain field. A macroscopic modelling considers the material as a two phases mixture. A crystallographical approach deals with granular aspects and existence of several variants of martensite. A moving boundaries description seems closer to physical mechanisms responsible to the deformation.

\subsection{Two phases approximation}

This kind of description is very useful in metallurgical and phenomenological approaches. Only averaging properties are taken into account. During the transformation, an initial volume $V$ of austenite is composed with a volume $V_{M}$ of martensite and a volume $V_{A}$ of austenite. Volume fraction of the product phase $\mathrm{f}=\mathrm{V}_{\mathrm{M}} / \mathrm{V}$ is an important parameter. Considering the transformation strain field has a zero value outside $V_{M}$ transforms equation (7) into :

$$
E_{i j}^{T}=\frac{V_{M}}{V} \frac{1}{V_{M}} \int_{V_{M}} \varepsilon_{i j}^{T}(r) d V=f \bar{\varepsilon}_{i j}^{T}
$$

This leads to define a second major parameter denoted by $\bar{\varepsilon}^{\mathrm{T}}$ which is the mean value of the transformation strain field over the martensitic domain. These two parameters $f$ and $\bar{\varepsilon}^{\top}$ allow to describe most of the kinematical aspects associated to the martensitic transformation. Time derivative of equation (8) gives :

$$
\dot{\mathrm{E}}_{\mathrm{ij}}^{\mathrm{T}}=\overleftarrow{\varepsilon}_{\mathrm{ij}}^{\mathrm{T}} \dot{\mathrm{f}}+\mathrm{f} \dot{\bar{\varepsilon}}_{\mathrm{ij}}^{\mathrm{T}}
$$

From such expression three macroscopic strain mechanisms are defined :

- Transformation without strain $\left(\dot{\mathrm{f}} \neq 0\right.$ and $\dot{\mathrm{E}}^{\mathrm{T}}=0$ ) characterized by : 


$$
\dot{E}_{\mathrm{ij}}^{\mathrm{T}}=\bar{\varepsilon}_{\mathrm{ij}}^{\mathrm{T}} \dot{\mathrm{f}}+\mathrm{f} \dot{\bar{\varepsilon}}_{\mathrm{ij}}^{\mathrm{T}}=0 \quad \text { when } \quad \bar{\varepsilon}^{\mathrm{T}}=0 \text { and } \quad \dot{\bar{\varepsilon}} \mathrm{T}=0
$$

From a mathematical point of view, this mechanism is only possible if the transformation occurs without any volume change, but physical consideration associated to the very low value of such volume change in shape memory alloys leads to consider this mechanism as associated to the production of a selfaccommodated morphology.

- Strain without transformation $\left(\dot{\mathrm{E}}^{\mathrm{T}} \neq 0\right.$ but $\left.\dot{\mathrm{f}}=0\right)$

$$
\dot{\mathrm{E}}_{\mathrm{ij}}^{\mathrm{T}}=\mathrm{f} \dot{\bar{\varepsilon}}_{\mathrm{ij}}^{\mathrm{T}}
$$

That is associated to the reorientation process occurring in the martensitic state.

- Transformation with strain.

$$
\dot{\mathrm{E}}_{\mathrm{ij}}^{\mathrm{T}}=\bar{\varepsilon}_{\mathrm{ij}}^{\mathbf{T}} \dot{\mathbf{f}}
$$

Very often by lack of an evolution law for $\bar{\varepsilon}^{T}$ this last relation is used to model the superelastic behavior. But assuming $\bar{\varepsilon}^{\mathrm{T}}$ remains constant along the loading path constitute a strong assumption as it is verified in section 6 of this paper.

Major kinematical difficulty related to this macroscopic description arises from the definition of kinematical constraints applied on the two parameters f and $\bar{\varepsilon}^{T}$. Due to their physical meaning, these quantities must satisfy some physical limitations. It is obvious that :

$$
0 \leq \mathrm{f} \leq 1
$$

But conditions on $\bar{\varepsilon}$ Tre difficult to define without additionnal assumptions. For instance one may postulate the existence of a maximal value $\varepsilon_{\max }$ for the equivalent mean transformation strain, assuming a symmetrical behavior (in the Von Mises meaning) leads to :

$$
0 \leq \frac{2}{3} \bar{\varepsilon}_{\mathrm{ij}}^{\mathrm{T}} \bar{\varepsilon}_{\mathrm{ij}}^{\mathrm{T}} \leq \varepsilon_{\max }^{2}
$$

In this relation, volume change due to the transformation is neglected that seems reasonable but difficulties come from the definition of $\varepsilon_{\max }$ and to the experimentally established non symmetrical behavior. These drawbacks disappear using the crystallographical approach.

\subsection{Crystallographical approach}

At this microstructural level, a transformation strain is related to each variant of martensite using the crystallographical theories [7]. Orientation of the transformation strain $\varepsilon^{n}$ associated to a given variant is defined from its habit plane normal $\mathbf{n}$ and its direction of transformation $\mathbf{m}$ (figure 4).

$$
\varepsilon_{\mathrm{ij}}^{\mathrm{n}}=\frac{1}{2} \mathrm{~g}\left(\mathrm{n}_{\mathrm{i}} \mathrm{m}_{\mathrm{j}}+\mathrm{n}_{\mathrm{j}} \mathrm{m}_{\mathrm{i}}\right)
$$

All variants have the same strain amplitude g. Considering $\varepsilon^{\mathrm{T}}(\mathrm{r})$ as a piecewise uniform function (figure 5) gives the following expression for the overall transformation strain (7):

$$
\mathbf{E}_{\mathrm{ij}}^{\mathrm{T}}=\frac{1}{V} \int_{V} \varepsilon_{\mathrm{ij}}^{\mathrm{n}} \theta^{\mathrm{n}}(\mathrm{r}) \mathrm{dV}=\sum_{\mathrm{n}} \varepsilon_{\mathrm{ij}}^{\mathrm{n}} \mathrm{f}^{\mathrm{n}} \quad \text { with } \theta^{\mathrm{n}}(\mathrm{r})=1 \text { if } \mathrm{r} \in \mathrm{V}^{\mathrm{n}}\left(=0 \text { if } \mathrm{r} \notin \mathrm{V}^{\mathrm{n}}\right)
$$

where $\mathrm{V}^{\mathrm{n}}$ denotes the volume occupied by a given variant. In this approach microstructural aspects are described using the volume fraction $\left(\mathrm{f}^{\mathrm{n}}=\mathrm{V}^{\mathrm{n}} / \mathrm{V}\right)$ of each variant as parameters. Physical limitations on $\mathrm{f}^{\mathrm{n}}$ are obviously determined as in the previous section. 


$$
\mathrm{f}^{\mathrm{n}} \geq 0 \quad \text { and } \quad \sum_{\mathrm{n}} \mathrm{f}^{\mathrm{n}}=\mathrm{f} \leq 1
$$

This description also represents the different behaviors defined in the previous section. Advantages come because no additional assumption is require to define the kinematical aspects related to the phase change.

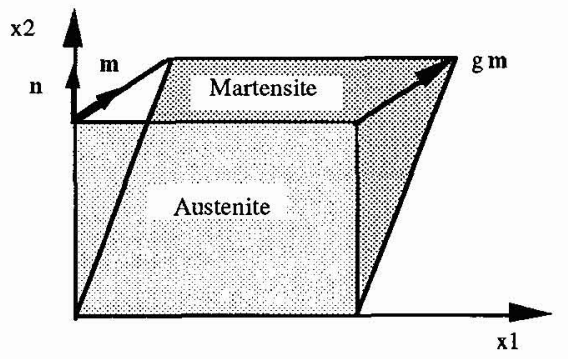

Figure 4: Local deformation induced by a Martensitic Transformation. Definition of the direction $\mathbf{m}$ of transformation and of the habit plane normal $\mathbf{n}$. The amplitude of the displacement in direction $\mathbf{m}$ is denoted by $\mathbf{g}$.
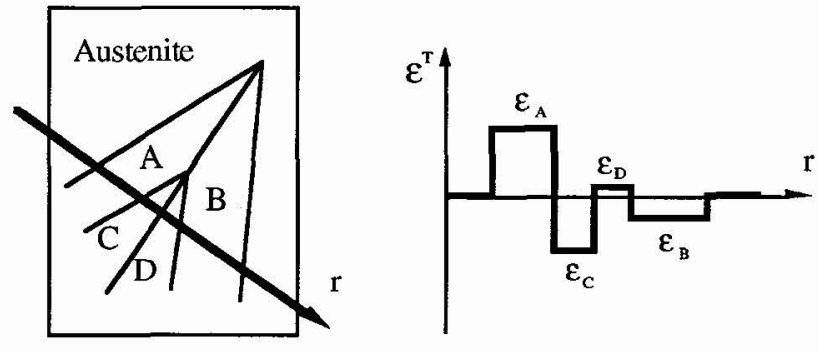

Figure 5: Transformation strain considered as a piecewise uniform function inside a polyvariants structure.

\subsection{Moving boundaries framework}

In any case, actual physical strain mechanisms responsible to the deformation in shape memory alloys are related to a boundary displacement, but this does not appear in the two previous descriptions. Assuming the transformation strain field suffer jumps along interfaces the transformation strain rate is expressed by [8]:

$$
\dot{\mathrm{E}}_{\mathrm{ij}}^{\mathrm{T}}=-\frac{1}{\mathrm{~V}} \int_{s}\left[\varepsilon_{\mathrm{ij}}^{\mathrm{T}}(\mathrm{r})\right] \omega_{\alpha}(\mathrm{r}) \mathrm{n}_{\alpha}(\mathrm{r}) \mathrm{ds}
$$

where $\left[\varepsilon^{\mathrm{T}}(r)\right]$ denotes the transformation jump along an interface oriented by a unit normal $\mathbf{n}$ and $\omega(\mathbf{r})$ is the velocity of these interfaces (figure 6). Inside the set of interfaces $S$ one may distinguish between the austenite-martensite boundaries (denoted by $\mathrm{s}_{\mathrm{am}}$ ) and the martensite-martensite one (denoted by $\mathrm{s}_{\mathrm{mm}}$ ). From such decomposition it comes :

$$
\dot{E}_{i j}^{T}=\frac{1}{V} \int_{S a m} \varepsilon_{i j}^{T}(r) \omega_{\alpha}(r) \eta_{\alpha}(r) d s-\frac{1}{V} \int_{S m m}\left(\varepsilon_{i j}^{T+}(r)-\varepsilon_{i j}^{T-}(r)\right) \omega_{\alpha}(r) \eta_{\alpha}(r) d s
$$

Both transformation and reorientation process appear in relation (19). The first mechanism is mostly related to the displacement of the $s_{a m}$ interfaces and the second one to that of the $s_{\mathrm{mm}}$. Such description is very closed to the physical mechanisms involved but unfortunately, difficulties arise from the determination of the velocity field $\omega(\mathrm{r})$ and from topological consideration about interface. In fine, the crystallographical approach seems more applicable.



Figure $6:$ Jump in the transformation strain field $\varepsilon^{\mathrm{T}}(\mathrm{r})$ across an interface $(\mathrm{s})$ oriented by a unit vector $\mathbf{n}$

Discontinuities on quantities $\mathrm{x}$ are denoted by $[\mathrm{x}]=(\mathrm{x})^{+}-(\mathrm{x})^{-}$; the velocity of interfaces is expressed by $\omega=\mathrm{d} \xi / \mathrm{dt}$ 


\section{THERMODYNAMICAL ASPECTS}

From these kinematical considerations thermodynamical analysis defines loading conditions to induce a martensitic transformation and derives the behavior for a representative element. This is obtained from the definition of a thermodynamical potential function of the control parameters (applied stress $\Sigma$ and temperature $\mathrm{T}$ ) and a set of internal variables related to the transformation strain field $\left\{\boldsymbol{\varepsilon}^{\mathrm{T}}(\mathbf{r})\right\}$. Let us consider a reference volume $\mathrm{V}$ (bounded by a surface $\partial \mathrm{V}$ ) of parent phase such as given surface forces applied on $\partial V$ are large enough to transform a volume $V_{M}$ into martensite. Complementary free energy $\Psi\left(\Sigma, T,\left\{\varepsilon^{T}(r)\right\}\right)$ is composed by elastic energy $W_{\text {elas }}$, potential energy $W_{\mathrm{T}}$ of the loading system, chemical energy $\mathcal{G}$ due to the phase transition and interfacial energy $\mathrm{W}_{\mathrm{s}}$.

$$
\Psi\left(\Sigma, \mathrm{T},\left\{\varepsilon^{\mathrm{T}}(\mathrm{r})\right\}\right)=-\left[\mathcal{G}+\mathrm{W}_{\text {elas }}+\mathrm{W}_{\mathrm{T}}+\mathrm{W}_{\mathrm{s}}\right]
$$

Chemical energy in thermoelastic martensitic transformation is only a function of the temperature. A linear approximation around $\mathrm{T}_{0}$, the thermodynamical equilibrium temperature is commonly used [9].

$$
\mathcal{G}(\mathrm{T})=\mathrm{B}\left(\mathrm{T}-\mathrm{T}_{0}\right) \mathrm{V}_{\mathrm{M}}
$$

Elastic energy is determined considering the local stress state $\sigma(r)$ composed by the applied stress and by an internal stress $\tau(\mathrm{r})$ related to the incompatibilities in the transformation strain field. Integration by parts and boundary conditions imposed on $\partial \mathrm{V}$ lead to the following expression for the elastic energy :

$$
W_{\text {elas }}=\frac{1}{2} \int_{v} \sigma_{i j}(r) \varepsilon_{i j}^{e}(r) d V=\frac{1}{2} V \Sigma_{i j} S_{i j k l} \Sigma_{k l}-\frac{1}{2} \int_{v} \tau_{i j}(r) \varepsilon_{i j}^{T}(r) d V
$$

Integral contribution in relation (22) defines an interaction energy related to the transformation strain field, in the following this important quantity is denoted by $\mathrm{W}_{\text {int }}$.

Potential energy due to the loading system is defined by :

$$
\mathrm{W}_{\mathrm{T}}=-\Sigma_{\mathrm{ij}}\left(\mathrm{S}_{\mathrm{ijkl}} \Sigma_{\mathrm{kl}}+\mathrm{E}_{\mathrm{ij}}^{\mathrm{T}}\right) \mathrm{V}
$$

Oblate shape of the martensite plates leads surface energy contribution negligible in regard to the elastic one. From (21), (22) and (23) complementary free energy per unit reference volume is expressed by :

$$
\Psi\left(\Sigma_{\mathrm{ij}}, \mathrm{T},\left\{\varepsilon^{\mathrm{T}}(\mathrm{r})\right\}\right)=\frac{1}{2} \Sigma_{\mathrm{ij}} \mathrm{S}_{\mathrm{ijkl}} \Sigma_{\mathrm{kl}}+\Sigma_{\mathrm{ij}} \mathrm{E}_{\mathrm{ij}}^{\mathrm{T}}-\mathrm{B}\left(\mathrm{T}-\mathrm{T}_{0}\right) \frac{\mathrm{V}_{\mathrm{M}}}{\mathrm{V}}+\frac{1}{2 \mathrm{~V}} \int_{\mathrm{v}} \tau_{\mathrm{ij}}(\mathrm{r}) \varepsilon_{\mathrm{ij}}^{\mathrm{T}}(\mathrm{r}) \mathrm{dV}
$$

Like for the kinematical study, different point of view may be used to describe microstructural aspects related to phase transition in relation (24). Two of these approaches are detailed in this paper. The more global one consider the material as a mixture of two phases. In that macroscopic approximation internal state of the material is described using only two internal variables. One is the volume fraction of product phase $\left(f=V_{M} / V\right)$, the other is the mean transformation strain $\dot{\varepsilon}^{T}$. In the Eshelby-Kröner framework, considering strain as piecewise uniform inside the two phases leads to the following result [10]:

$$
\Psi\left(\Sigma_{i j}, T, f, \bar{\varepsilon}_{i j}^{T}\right)=\frac{1}{2} \Sigma_{i j} S_{i j k l} \Sigma_{k l}+\Sigma_{i j} f \bar{\varepsilon}_{i j}^{T}-B\left(T-T_{0}\right) f+f(1-f) \bar{\varepsilon}_{i j}^{T} A_{i j k l} \bar{\varepsilon}_{k l}^{T}
$$

where $\mathrm{A}$ is a constant tensorial quantity related to elastic properties of the material and to the morphology of the product phase. From particular form of potential (25) an oblique line is defined for the behavior (related to the $f(1-f)$ contribution as reported by $[11,12]$ ) (figure 7). This is equivalent to consider that internal stress turns to zero in the martensitic state that is in contradiction with observations. It is possible to avoid such troubles using a more accurate description for the microstructure dealing with the polycrystalline nature of the material and with the formation of several variants of martensite inside grain. This is the object of the following section. 


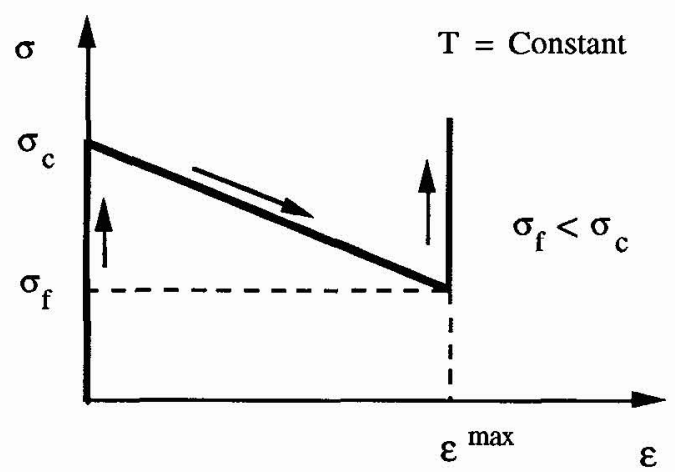

Figure 7 : Oblic line determined for superelasticity using a global two phases approximation to derive the thermodynamical potential (25).

\section{MICROMECHANICAL MODELLING}

Scale transition methods issued from field theory have been successfully applied to described the elastic or elastoplastic behavior of materials $[13,14]$. In the following, this framework is applied to phase transitions. Such approach needs to determine a local behavior law for an elementary representative volume and to define the integral equation associated to the microheterogeneous material. In this work, a selfconsistent approach is used to approximate this integral equation.

\subsection{Interaction matrix and local behavior law}

In polycrystal, grains are chosen as elementary representative volume to account with the granular structure and the existence of several variants of martensite. Each grain is considered as a single crystal and the crystallographical point of view presented in section 3.2 is used for the kinematical description.

Potential energy (24) is now defined at the grain level. In this relation, interaction energy $W_{\text {int }}$, that is a complex function of the transformation strain field, is the only contribution not totally described using the $\mathrm{f}^{\mathrm{n}}$ parameters as internal variables. A realistic evaluation for this energy is obtained from physical considerations on the growth of martensitic plates in stress-induced transformation. Some typical features are associated to the stress-induced transformation inside grains in polycrystalline material. Micrography 1.c shows that only a limited number of variants are formed. Each of them is growing inside a well-defined domain of the grain. Assuming most of internal stresses originate from martensite to martensite interactions a cluster description is used to model these microstructural aspects.

Let us consider inside a grain of volume $\mathrm{V}$, a domain $V_{n}$ filled by a given variant. During the transformation $V_{n}$ is composed by a mixture of martensite plates having the same orientation and with austenite (figure 8).


Figure 8: Microsctuctural aspects used into a cluster model to define interaction energy inside grain for a superelastic alloy. 
Three scalar parameters are used to characterize this microstructure.

$$
f^{n}=\frac{v_{n}}{V}=\frac{v_{n}}{V_{n}} \frac{V_{n}}{V}=\varphi^{n} F_{n}
$$

Volume fraction $\mathrm{f}^{\mathrm{n}}$ for variant is classically defined as in section 3.2. Inside each domain $\mathrm{V}_{\mathrm{n}}$ parameter $\varphi^{\mathrm{n}}$ characterizes the amount of martensite and mean value of the transformation strain field is defined as :

$$
\bar{\varepsilon}_{i j}^{n}=\frac{1}{V_{n}} \int_{V_{n}} \varepsilon_{i j}^{T}(r) d V=\varepsilon_{i j}^{n} \frac{v_{n}}{V_{n}}=\varepsilon_{i j}^{n} \varphi^{n}
$$

Using (27), considering the transformation strain field as piecewise uniform turns interaction energy into:

$$
W^{i n t}=-\frac{1}{2 V} \int_{V} \tau_{i j}(r) \varepsilon_{i j}^{T}(r) d V=-\frac{1}{2 V} \sum_{n} \bar{\tau}_{i j}^{n} \bar{\varepsilon}_{i j}^{n} V_{n}
$$

where $\bar{\tau}^{\mathrm{n}}$ denotes the mean value of the internal stress over volume $\mathrm{V}_{\mathrm{n}}$. Averaging over all the crystal gives the following property:

$$
\frac{1}{V} \int_{V} \tau_{i j}(r) E_{i j}^{T} d V=\frac{1}{V} \sum_{n} \tau_{i j}^{n} E_{i j}^{T} V_{n}=0
$$

From (28) and (29), considering domain $\mathrm{V}_{\mathrm{n}}$ as an ellipsoidal inclusion characterized by an Eshelby tensor $\mathcal{S}^{\mathrm{n}}$, gives :

$$
W^{\mathrm{int}}=\frac{1}{2} \sum_{\mathrm{n}}\left\{\bar{\varepsilon}_{\mathrm{ij}}^{\mathrm{n}}-\mathrm{E}_{\mathrm{ij}}^{\mathrm{T}}\right\} \mathrm{C}_{\mathrm{ijkl}}\left(\mathrm{I}_{\mathrm{klrs}}-S_{\mathrm{klrs}}^{\mathrm{n}}\right)\left\{\bar{\varepsilon}_{\mathrm{rs}}^{\mathrm{n}}-\mathrm{E}_{\mathrm{rs}}^{\mathrm{T}}\right\} \mathrm{F}_{\mathrm{n}}
$$

As only few variants are formed, energy (30) can be evaluated using a pair interaction form. In that case coming from strong correlations that exit between two adjacent domains (see micrography 1.c) the following properties are assumed $S^{p}=S^{q}=S p q$ and $\varphi^{p}=\varphi^{q}$, that give :

$$
W^{i n t}=\frac{1}{2} f^{P} f^{q}\left(\varepsilon_{i j}^{p}-\varepsilon_{i j}^{q}\right) C_{i j k l}\left(I_{k l r s}-S_{k l s s}^{p q}\right)\left(\varepsilon_{r s}^{p}-\varepsilon_{r s}^{q}\right)
$$

This energy depends of parameters $\mathrm{f}^{\mathrm{n}}$ but also of the domain shape and orientation. Take this complete set of internal parameters into the complementary free energy (24) is very complicated. A more practical way consists to define an interaction matrix from minimization of the $\left(\varepsilon^{p}-\varepsilon^{q}\right) C\left(I-S^{p q}\right)(\varepsilon p-\varepsilon q)$ contribution with respect to the orientation of tensor $S$ pq, assuming a constant aspect ratio.

This optimization defines two kinds of interactions in accordance with experimental observations. Weak interactions correspond to self-accommodated variants and strong interactions to incompatible plates. That gives :

$$
\mathrm{W}_{\mathrm{int}}=\frac{1}{2} \sum_{\mathrm{n}, \mathrm{m}} \mathrm{H}^{\mathrm{nm}} \mathrm{f}^{\mathrm{n}} \mathrm{f}^{\mathrm{m}}
$$

In fine, variables $\mathrm{f}^{\mathrm{m}}$ are sufficient to describe evolution of the microstructural state. Thermodynamical potential is now expressed at the crystal level :

$$
\Psi\left(\Sigma_{\mathrm{ij}}, \mathrm{T}, \mathrm{f}^{\mathrm{n}}\right)=\frac{1}{2} \Sigma_{\mathrm{ij}} \mathrm{S}_{\mathrm{ijkl}} \Sigma_{\mathrm{kl}}+\Sigma_{\mathrm{ij}} \sum_{\mathbf{n}} \varepsilon_{\mathrm{ij}}^{\mathrm{n}} \mathrm{f}^{\mathrm{n}}-\mathrm{B}\left(\mathrm{T}-\mathrm{T}_{0}\right) \sum_{\mathbf{n}} \mathrm{f}^{\mathrm{n}}+\frac{1}{2} \sum_{\mathrm{n}, \mathrm{m}} \mathrm{H}^{\mathrm{nm}} \mathbf{f}^{\mathrm{n}} \mathrm{f}^{\mathrm{m}}
$$


Existence of kinematical constraints (17) on the internal variables used imposes to introduce a Lagrangian functional $L\left(\Sigma_{\mathrm{ij}}, \mathrm{T}, \mathrm{f}^{\mathrm{n}}\right)$ to derive the driving forces $\mathrm{F}^{\mathrm{n}}$ associated to internal variable $\mathrm{f}^{\mathrm{n}}$ [15].

$$
\mathcal{L}\left(\Sigma_{\mathrm{ij}}, \mathrm{T}, \mathrm{f}^{\mathrm{n}}\right)=\Psi\left(\Sigma_{\mathrm{ij}}, \mathrm{T}, \mathrm{f}^{\mathrm{n}}\right)-\lambda_{0}\left[\sum_{\mathrm{n}} \mathrm{f}^{\mathrm{n}}-1\right]-\sum_{\mathbf{n}} \lambda_{\mathrm{n}}\left[-\mathrm{f}^{\mathrm{n}}-0\right]
$$

Lagrange multipliers $\lambda_{0}$ and $\lambda_{\mathrm{n}}$ used in (34) are positive quantities associated with inequality constraints $\mathrm{f} \leq 1$ and $\left(-\mathrm{f}^{\mathrm{n}}\right) \leq 0$. Definition of the driving force turns into:

$$
F^{n}=-B\left(T-T_{0}\right)+\Sigma_{i j} \varepsilon_{i j}^{n}+\sum_{m} H^{n m} f^{m}+\lambda_{n}-\lambda_{0}
$$

Hysteretic behavior is determined considering variant growth (or shrink) occurs when driving force $\mathrm{F}^{\mathrm{n}}$ reaches a critical value. This resistive dissipative force $F_{c}$ is assumed to be equal for all the variants. Conditions necessaries to obtain a transformation flow are established like:

$$
\begin{array}{llll}
\dot{\mathrm{f}}^{\mathrm{n}}=0 & \text { if } & \mathrm{F}^{\mathrm{n}}<\mathrm{F}_{\mathrm{c}} & \forall \dot{\mathrm{F}}_{\mathrm{c}} \\
\dot{\mathrm{f}}^{\mathrm{n}}=0 & \text { if } & \mathrm{F}^{\mathrm{n}}=\mathrm{F}_{\mathrm{c}} & \text { and } \dot{\mathrm{F}}_{\mathrm{c}}=0 \\
\dot{\mathrm{f}}^{\mathrm{n}} \neq 0 & \text { if } & \mathrm{F}^{\mathrm{n}}=\mathrm{F}_{c} & \text { and } \dot{\mathrm{F}}_{\mathrm{c}}=\dot{\mathrm{F}}^{\mathrm{n}}
\end{array}
$$

where $\mathrm{F}_{\mathrm{c}}$ is a positive material constant. Second law of the thermodynamics and the energy balance require:

$$
\dot{\Psi}_{\mid \Sigma, T}=\sum_{\mathbf{n}} F^{n} \dot{\mathbf{I}}^{\mathrm{n}}=\dot{\mathrm{W}}^{\mathrm{d}} \geq 0
$$

Assuming the dissipation occurs without any coupling effect on each variant, forward and reverse transformations are now controlled by :

$$
A->M \Rightarrow F^{n} \dot{\mathrm{f}}^{n}=F_{c} \dot{\mathbf{f}}^{\mathrm{n}} \quad \text { and } \quad \mathrm{M} \rightarrow \mathrm{A} \Rightarrow \mathrm{F}^{\mathrm{n}} \dot{\mathrm{f}}^{\mathrm{n}}=-\mathrm{F}_{\mathrm{c}} \dot{\mathrm{f}}^{\mathrm{n}}
$$

From expression (35) and conditions (38), each variant has to follow a local transformation criterion, defined in the forward and reverse case.

$$
-\mathrm{B}\left(\mathrm{T}-\mathrm{T}_{0}\right)+\Sigma_{\mathrm{ij}} \varepsilon_{\mathrm{ij}}^{\mathrm{n}}+\sum_{\mathrm{m}} \mathrm{H}^{\mathrm{nm}} \mathrm{f}^{\mathrm{m}}-\lambda_{\mathrm{n}}+\lambda_{0}={ }_{-}^{+} \mathrm{F}_{\mathrm{c}}
$$

Kinematical conditions (17) impose to verify $n+1$ additional relations:

$$
\begin{gathered}
\lambda_{\mathrm{n}}=-\mathrm{B}\left(\mathrm{T}-\mathrm{T}_{0}\right)+\Sigma_{\mathrm{j} j} \varepsilon_{\mathrm{ij}}^{\mathrm{n}}+\sum_{\mathrm{m}} \mathrm{H}^{\mathrm{nm}} \mathrm{f}^{\mathrm{m}}+\lambda_{0}{ }_{-}^{+} \mathrm{F}_{\mathrm{c}} \geq 0 \\
\lambda_{0}=\mathrm{B}\left(\mathrm{T}-\mathrm{T}_{0}\right)-\Sigma_{\mathrm{ij}} \varepsilon_{\mathrm{ij}}^{\mathrm{n}}-\sum_{\mathrm{m}} \mathrm{H}^{\mathrm{nm}} \mathrm{f}^{\mathrm{m}}-\lambda_{\mathrm{n}}{ }_{-}^{+} \mathrm{F}_{\mathrm{c}} \geq 0
\end{gathered}
$$

When criterion (39) and conditions (40) are satisfied, evolution law for parameter $\mathrm{f}^{\mathrm{n}}$ is determined using the consistency condition. This gives the following constitutive equation for the transformation strain rate applied to a unit volume of crystal.

$$
\dot{\varepsilon}_{\mathrm{ij}}^{\mathbf{T}}=\sum_{\mathrm{n}} \varepsilon_{\mathrm{ij}}^{\mathrm{n}} \sum_{\mathrm{m}}\left(\mathbf{H}^{\mathrm{nm}}\right)^{-1}\left[\varepsilon_{\mathrm{kl}}^{\mathrm{m}} \dot{\Sigma}_{\mathrm{kl}}-\mathbf{B} \dot{\mathrm{T}}\right]
$$

Set of equations $(39,40,41)$ characterizes the transformation plasticity in the single crystal case (figure 9). 




Figure 9 : Grain behavior used as local constitutive equation for polycrystalline material.

\subsection{Micro-macro transition}

Due to the existence of an internal stress field arising from the granular structure an accurate determination of a macroscopic constitutive equation like (41) is impossible in the polycrystalline case. Homogenization methods help us to overcome this difficulty [16]. Fundamental local basic relations used are the local equilibrium condition, the continuity of deformation and the local behavior law (41). Local quantities must be consistent with boundary conditions. In the following capital letters are used for overall quantities and lower case for local ones (figure 10). In this work, temperature $\mathrm{T}$ is considered as uniform. We consider the behavior (41) on a dual form including the thermoelastic contributions $\left(\dot{\varepsilon}=\dot{\varepsilon}^{\mathrm{e}}+\dot{\varepsilon}^{\text {th }}+\dot{\varepsilon}^{\mathrm{T}}\right)$ :

$$
\dot{\sigma}_{\mathrm{ij}}(r)=l_{\mathrm{ijkl}}(r) \dot{\varepsilon}_{\mathrm{kl}}(\mathrm{r})-\mathrm{m}_{\mathrm{ij}}(\mathrm{r}) \dot{\mathrm{T}}
$$

Local modulus $1(r)$ and $m(r)$ are expressed from their deviation part to a homogeneous reference medium (having uniform tangent modulus $\mathrm{L}^{\circ}$ and $\mathrm{M}^{\circ}$ ). This medium is subjected to a uniform strain rate $\dot{\mathrm{E}}^{\circ}$.

$$
l_{\mathrm{ijkl} l}(\mathrm{r})=\mathrm{L}_{\mathrm{ijkl}}^{\mathrm{o}}+\delta \mathrm{l}_{\mathrm{ijkl}}(\mathrm{r}) \text { and } \mathrm{m}_{\mathrm{ij}}(\mathrm{r})=\mathrm{M}_{\mathrm{ij}}^{\mathrm{o}}+\delta \mathrm{m}_{\mathrm{jj}}(\mathrm{r})
$$

Equilibrium condition gives :

$$
\mathrm{L}_{\mathrm{ijkl}}^{\mathrm{o}}\left(\dot{\varepsilon}_{\mathrm{k} 1}(\mathrm{r})\right)_{\mathrm{j} j}+\left(\delta \mathrm{l}_{\mathrm{ijkl}}(\mathrm{r}) \dot{\varepsilon}_{\mathrm{kl}}(\mathrm{r})-\delta \mathrm{m}_{\mathrm{ij}}(\mathrm{r}) \dot{\mathrm{T}}_{\mathrm{j}}=0\right.
$$

Using the Green tensor method, the following integral equation is obtained [17]:

$$
\dot{\varepsilon}_{m n}(r)=\dot{E}_{m n}^{o}+\int_{v} \Gamma_{m n i j}^{o}\left(r-r^{\prime}\right)\left[\delta l_{i j k l}\left(r^{\prime}\right) \dot{\varepsilon}_{k l}\left(r^{\prime}\right)-\delta m_{i j}\left(r^{\prime}\right) \dot{T}\right] d V^{\prime}
$$

where $\Gamma^{\circ}$ denotes the modified Green tensor. According to Kröner [18] this tensor is composed with a local component $\Gamma^{\mathrm{ol}}$ and a non local one $\Gamma^{\mathrm{nl}}$. This property turns integral equation (45) into :

$\dot{\varepsilon}_{m n}(r)=\dot{E}_{m n}^{o}+\Gamma_{m n i j}^{o l}(r)\left[\delta l_{i j k l}(r) \dot{\varepsilon}_{k l}(r)-\delta m_{i j}(r) \dot{T}\right]+\int_{v} \Gamma_{m n j j}^{n l}\left(r-r^{\prime}\right)\left[\delta l_{i j k l}\left(r^{\prime}\right) \dot{\varepsilon}_{k l}\left(r^{\prime}\right)-\delta m_{i j}\left(r^{\prime}\right) \dot{T}\right] d V^{\prime}$

where $\delta\left(r-r^{\prime}\right)$ is a Dirac function. All difficulties relevant to the resolution of equation (46) are located in the determination of its last contribution. This problem is avoid using a particular homogeneous reference medium such as the following condition is satisfied:

$$
\left.\int_{V}\left[I_{i j k l}(r)-L_{i j k l}^{o}\right] \dot{\varepsilon}_{k l}(r)-\left[m_{i j}(r)-M_{i j}^{o}\right] \dot{T}\right) d V=0
$$


In this case, the last contribution in relation (46) becomes negligible. From (47) $\mathrm{L}^{\circ}$ and $\mathrm{M}^{\circ}$ must verify :

$$
\int_{\mathrm{v}}\left(\mathrm{l}_{\mathrm{ijkl}}(\mathrm{r}) \dot{\varepsilon}_{\mathrm{kl}}(\mathrm{r})-\mathrm{m}_{\mathrm{ij}}(\mathrm{r}) \dot{\mathrm{T}}\right) \mathrm{dV}-\mathrm{L}_{\mathrm{ijkl}}^{\mathrm{o}} \int_{\mathrm{v}} \dot{\varepsilon}_{\mathrm{kl}}(\mathrm{r}) \mathrm{dV}+\mathrm{M}_{\mathrm{ij}}^{\mathrm{o}} \dot{\mathrm{T}}=0
$$

That is equivalent to :

$$
\dot{\Sigma}_{\mathrm{ij}}=\mathrm{L}_{\mathrm{ijkl}}^{\mathrm{o}} \dot{\mathrm{E}}_{\mathrm{kl}}-\mathrm{M}_{\mathrm{ij}}^{\mathrm{o}} \dot{\mathrm{T}}
$$

Particular choice (47) leads that the homogeneous reference medium used is equal to the effective medium to be determined.

$$
\mathrm{L}_{\mathrm{ijkl}}^{\circ}=\mathrm{L}_{\mathrm{ijkl}}^{\text {eff }} \quad ; \quad \mathrm{M}_{\mathrm{ij}}^{\mathrm{o}}=\mathrm{M}_{\mathrm{ij}}^{\mathrm{eff}} \quad \text { and } \Gamma_{\mathrm{ijkl}}^{\mathrm{ol}}=\Gamma_{\mathrm{ijkl}}^{\text {eff }}
$$

Relation (46) turns now into

$$
\dot{\varepsilon}_{\mathrm{mn}}(\mathrm{r})=\dot{\mathrm{E}}_{\mathrm{mn}}^{\mathrm{o}}+\Gamma_{\mathrm{mnij}}^{\mathrm{eff}}(\mathrm{r})\left[\left(\mathrm{l}_{\mathrm{ijkl}}(\mathrm{r})-\mathrm{L}_{\mathrm{ijkl}}^{\mathrm{eff}}\right) \dot{\varepsilon}_{\mathrm{kl}}(\mathrm{r})-\left(\mathrm{m}_{\mathrm{ij}}(\mathrm{r})-\mathrm{M}_{\mathrm{ij}}^{\mathrm{eff}}\right) \dot{\mathrm{T}}\right]
$$

Two concentration tensors that relate local quantities to global ones are finally defined.

$$
\begin{aligned}
& A_{\text {mnkl }}(r)=\left[I_{m n k l}-\Gamma_{m n i j}^{e f f}(r)\left[\left(l_{i j k l}(r)-L_{i j k l}^{e f f}\right)\right]^{-1}\right. \\
& a_{k l}(r)=A_{k I m n}(r) \Gamma_{m n i j}^{e f f}(r)\left[m_{i j}(r)-M_{i j}^{e f f}\right]
\end{aligned}
$$

These quantities allow to determine the uniform tangent modulus $\mathrm{L}^{\text {eff }}$ and $\mathrm{M}^{\mathrm{eff}}$ from the knowledge of the local one derived from relation (42).

$$
L_{i j k l}^{e f f}=\int_{v} 1_{i j m n}(r) A_{n n k l}(r) d V \quad \text { and } \quad M_{i j}^{e f f}=\int_{v}\left[1_{i j k l}(r) a_{k l}(r)-m_{i j}(r)\right] d V
$$

Considering the polycrystalline material as an aggregate of grains having a homogeneous behavior, leads the concentration tensors and the local tangent modulus to be piecewise uniform. This turns (54) into :

$$
L_{i j k l}^{e f f}=\sum_{N} l_{i j m n}^{N} A_{m n k l}^{N} F^{N}
$$

where $\mathrm{F}^{\mathrm{N}}$ denotes the volume fraction of the grain family with label N. In this description each grain is characterized by its crystallographical orientation (obtained by X-ray measurement), its shape (through $\Gamma^{\text {ol }}$ ) and its volume fraction $\mathrm{F}^{\mathrm{N}}$. Overall constitutive relation for polycrystal is now completely defined from the internal structure evolution (represented in this approach using the volume fraction associated to the different variants of martensite). Intergranular features (grain missorientation ...) are derived from the micro-macro transition scheme. The local behavior law defined in the previous section is used to account for the intragranular aspects (as multivariant transformation).

Overall variables

$\begin{array}{cc} & \Sigma \\ \text { L }^{\text {eff }} & \text { E } \\ \text { M }^{\text {ff }} & T\end{array}$

Local variables

$$
\begin{aligned}
& \sigma(r)=\sum_{N} \sigma^{N} \theta^{N}(r) \\
& \varepsilon(r)=\sum_{N} \varepsilon^{N} \theta^{N}(r) \\
& l(r)=\sum_{N} 1^{N} \theta^{N}(r)
\end{aligned}
$$

Figure 10 : Local and global parameters used in the scale transition framework.

Pollycristalline material is described using a set of $\mathrm{N}$ grains having piecewise uniform stress, strain and local modulus. 


\section{APPLICATIONS}

Numerical results obtained for the superelastic behavior of $\mathrm{Cu}-\mathrm{Zn}$ - $\mathrm{Al}$ shape memory alloys are presented in this section. The scale transition method used in this approach needs to characterize both the polycrystalline structure and the martensitic transformation.

Elastic behavior of the material, supposed to be isotropic and homogeneous, is defined by the shear modulus $(\mu \approx 40 \mathrm{GPa})$ and the Poisson ratio $(v \approx 0.3)$.

Polycrystalline structure is described using a set of grains having a given shape and orientation, and occupying a well-defined volume fraction of the material. These characteristics are supposed to remain constant along the transformation. All these data can be experimentally determined. In this work, granular aspects are described in a simplified way using spherical grains and isotropic texture with a set of 100 grains.

In $\mathrm{Cu}-\mathrm{Zn}-\mathrm{Al}$ Shape memory alloys, twenty-four variants of martensite, forming six self-accommodated groups, are observed. Habit plane normal and transformation direction are of [2 11 12] type [19]. Transformation displacement $\mathrm{g}$ is about 0.23 . Parameter $\mathrm{B}$ used in the thermodynamical potential (33) is experimentally obtained from tensile test performed on single crystal $\left(B \approx 0.23 \mathrm{MPa} \cdot \mathrm{K}^{-1}\right)$. Critical force $\mathrm{F}_{\mathrm{c}}$ that appears in conditions (36) is deduced from measurement of the transformation temperatures in the parent phase $\left(\mathrm{M}_{\mathrm{s}}\right.$ and $\left.\mathrm{A}_{\mathrm{f}}\right)$. In these alloys micromechanical determination of the interaction matrix $\mathrm{H}^{\mathrm{nm}}$ gives two kinds of terms, weak one $(\mu / 1000)$ for self-accommodated variants and strong one $(\mu / 150)$ for the other.

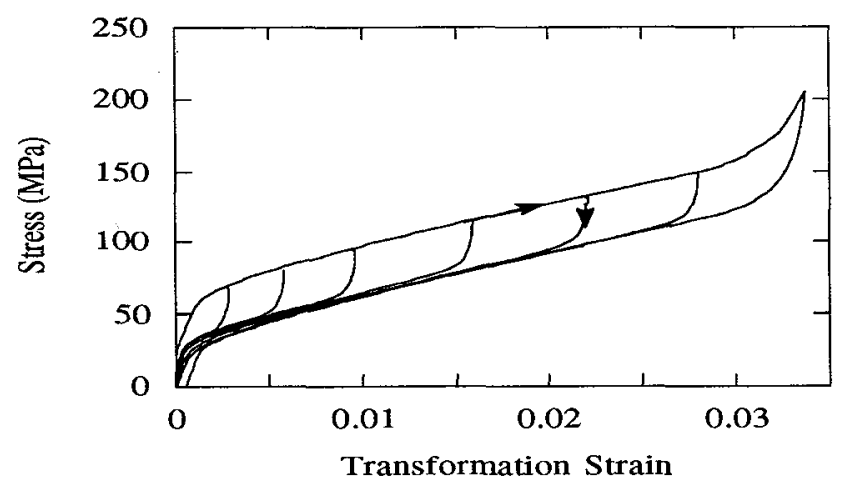

Figure 11: Overall superelastic behavior determined using a scale transition method.

Uniaxial tensile test on a Cu-Zn-Al Shape Memory Alloy (at $\mathrm{T}=20^{\circ} \mathrm{C}$ with $\mathrm{M}_{S}=10^{\circ} \mathrm{C}$ and $\mathrm{A}_{\mathrm{f}}=15^{\circ} \mathrm{C}$.)

Figure 11 illustrates the overall superelastic behavior computed by this way for a uniaxial tensile test at room temperature. Particular $\mathrm{Cu}-\mathrm{Zn}-\mathrm{Al}$ alloy used is characterized from its transformation temperatures $\mathrm{M}_{\mathrm{s}}$ and $\mathrm{A}_{\mathrm{f}}$, respectively taken as $10^{\circ} \mathrm{C}$ and $15^{\circ} \mathrm{C}$. The maximal overall transformation strain thus determined is around three per cent, what is realistic for this kind of alloy.

These results are consistent with experimental superelastic data published by Vacher and Lexcellent [20] on a Cu- $\mathrm{Zn}-\mathrm{Al}$ shape memory alloy $\left(\mathrm{M}_{\mathrm{s}}=-97^{\circ} \mathrm{C}\right.$ and $\left.\mathrm{A}_{\mathrm{f}}=-91^{\circ} \mathrm{C}\right)$.

Uniaxial tensile test performed at $\mathrm{T}=-80^{\circ} \mathrm{C}$ shows that transformation stress, transformation slope and hysteresis size are correctly evaluated by the model (figure 12). From these experimental data it is observed that a variation in the test temperature is quantitatively described by the micromechanical approach used (figure 12). It is worth to notice that these results are obtained without assistance of adjustable parameter.

Evolution of the macroscopic internal variables ( $f$ and $\bar{\varepsilon}^{T}$ ) used in the global two phases approach presented in sections 3.1 and 4 are also computed. Comparison with resistivity measurement performed by Vacher and Lexcellent [20] in parallel to their tensile tests shows that the stress-induced transformation kinetics is correctly described (figure 13). Numerical result presented at figure 14 established that the global mean transformation strain is a non constant parameter presenting a very strong decrease in the first stage of the 
transformation. This evolution is never taken into account in phenomenological model that consider it as a constant value by lack of an evolution law. One advantage of the approach employed here is to compute such a law for a given loading mode.

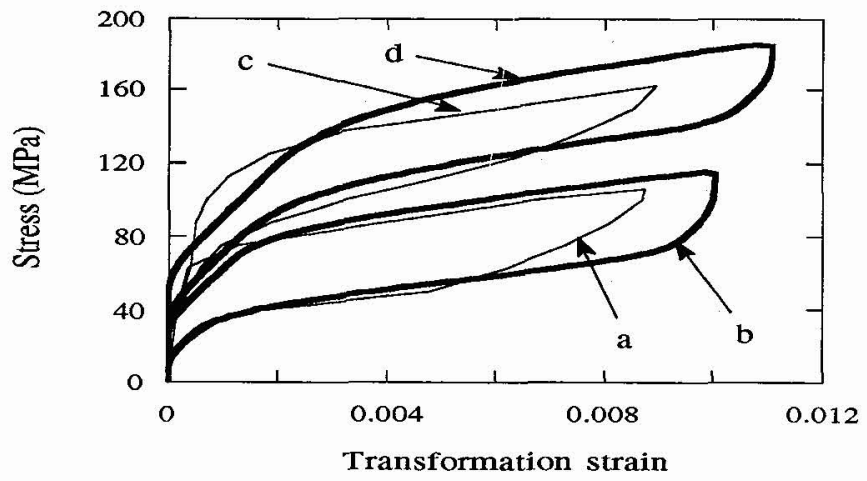

Figure 12: Influence of the temperature on the superelastic behavior. Comparison between experimental results (a and c) [20] and self-consistent determination (b and d). $\mathrm{T}=-80^{\circ} \mathrm{C}$ for $\mathrm{a}$ and $\mathrm{b} ; \mathrm{T}=-70^{\circ} \mathrm{C}$ for $\mathrm{c}$ and $\mathrm{d}$.

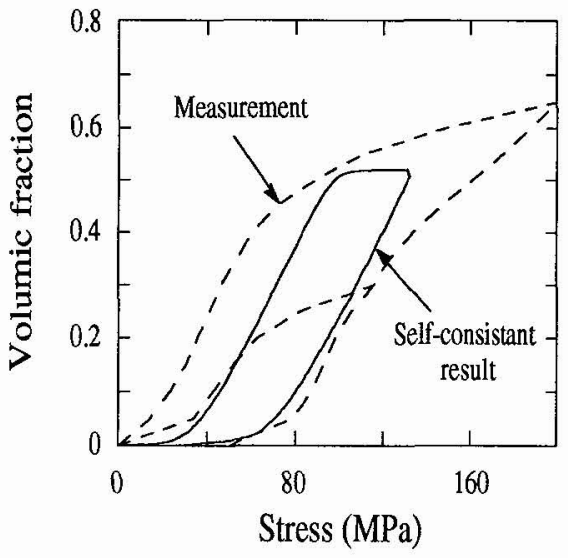

Figure 13: Kinetics of a stress-induced transformation. Comparison between experimental results [20] and self-consistent determination for tensile test. $\mathrm{T}=-80^{\circ} \mathrm{C}\left(\mathrm{M}_{\mathrm{S}}=-97^{\circ} \mathrm{C} \mathrm{A}_{\mathrm{f}}=-91^{\circ} \mathrm{C}\right)$.

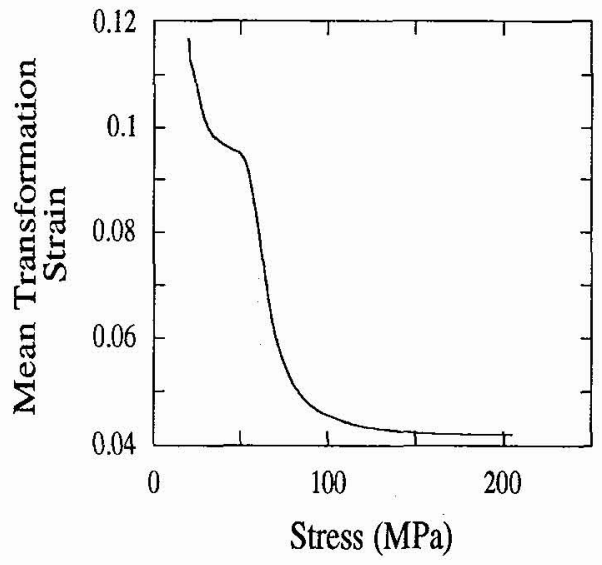

Figure 14: Evolution of the mean Transformation Strain for tensile test condition.

\section{APPLICATION TO STRUCTURE CALCULATION}

The micromechanical approach presented in this paper deals successfully with non symmetrical aspect related to the superelastic behavior in shape memory alloys. This phenomenon comes from crystallographical features related to the martensitic transformation as established in [4]. At the crystal level variants of stress-induced martensite are different with respect to the loading condition. In consequence, well-oriented variants have not the same occurrence for tensile test and compressive one in polycrystalline material. This experimentally observed dissymmetrical behavior [20] is well captured by the proposed model (figure 15). Like for experiments on $\mathrm{Cu}-\mathrm{Zn}-\mathrm{Al}$ alloys, critical transformation stress, transformation slope and hysteresis size are larger in compressive test than in tensile one. These tendencies are numerically obtained without any additional assumption, using an isotropic crystallographical texture. 


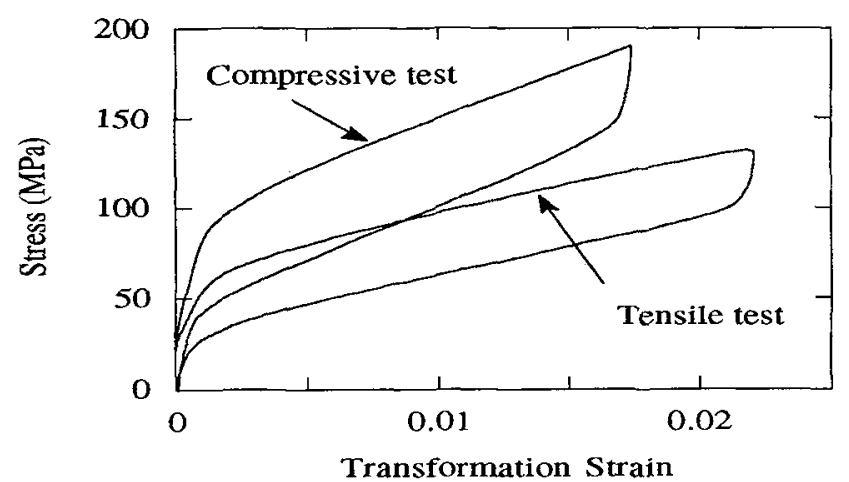

Figure 15 : Dissymmetry obtained for tensile and compressive uniaxial test using the micromechanical approach.

Applications for other loading conditions determine a non symmetrical transformation surface (figure 16) [4]. Such surface is very far from the Von Mises criterion. It can be noticed that the computed surface kept the first bisector as symmetrical axis.

This determination is very useful for structure calculation. Self-consistent resolution gives no analytical flow rule at the macroscopic level. That is a limitation for application to structure calculation. Phenomenological considerations about the shape of the transformation surface presented at figure 16 allow to overcome this difficulty. As the material is considered as an isotropic medium a macroscopic transformation criterion is expressed using the scalar invariants of the stress tensor. In shape memory alloys hydrostatic pressure has a very little influence on the martensitic transformation. Thus only second and third scalar invariant of the deviatoric stress (resp. $J_{2}$ and $J_{3}$ ) are used, that is obtained as for example in the Prager equation.

$$
F\left(J_{2}, J_{3}, \varepsilon^{T}, T\right)=J_{2}\left[1+b \frac{J_{3}}{J_{2}^{3 / 2}}\right]-K^{2}\left(\varepsilon^{T}, T\right)=0
$$

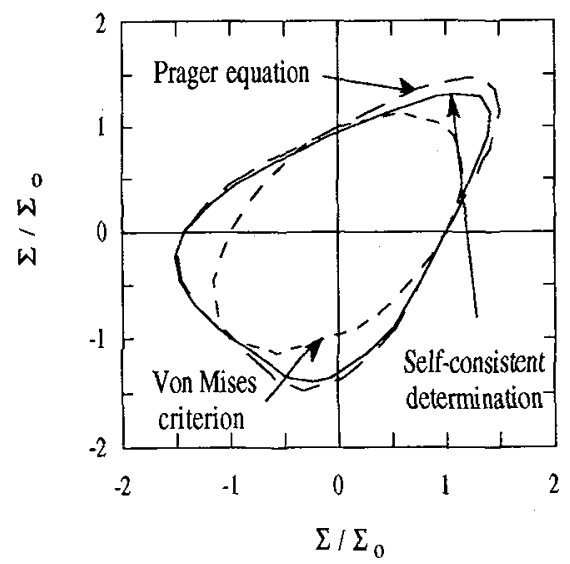

Figure 16: Transformation surface determined in a two-dimensional stress space using the self-consistent framework. Comparison with surfaces defined from the Von Mises criterion and the Prager equation [4].

Due to the thermomechanical characteristics of the shape memory behavior, criterion (56) is a function of the temperature. By analogy with plasticity, the inelastic strain $\varepsilon^{\mathbf{T}}$ is chosen as hardening parameter. 
Identification with tensile and compressive test (denoting by $\sigma_{\mathrm{t}}$ and $\sigma_{\mathrm{c}}$ the critical transformation stresses in each loading condition) determines the following expressions for parameter $\mathrm{b}$ and function $\mathrm{K}\left(\varepsilon^{\mathrm{T}}, \mathrm{T}\right)$.

$$
\mathrm{b}=\frac{\sqrt{27}}{2} \frac{\sigma_{\mathrm{c}}^{2}-\sigma_{\mathrm{t}}^{2}}{\sigma_{\mathrm{c}}^{2}+\sigma_{\mathrm{t}}^{2}} \quad \mathrm{~K}^{2}\left(\varepsilon^{\mathrm{T}}, \mathrm{T}\right)=\frac{\sigma_{\mathrm{t}}}{\sqrt{3}} \sqrt{1+\frac{2 \mathrm{~b}}{\sqrt{27}}}
$$

Loading surface such obtained shows a very good accordance with the micro-macro computation (figure 16). This non symmetrical transformation criterion is applied to determine an analytical behavior law very useful for structure calculation. Such equation is derived using the normality rule. Good results are obtained for the bending behavior of superelastic beam [6].

\section{CONCLUSION}

Numerical results obtained from the micromechanical model presented in this paper are in good agreement with experimental observations. These results are obtained without assistance of any fitting parameter. All input data can be experimentally determined. Such approach gives information on the microstructure evolution during the loading process and can be successfully applied to complex loading conditions.

The proposed model well represents the dissymmetry experimentally observed between tensile and compressive test on superelastic alloys. Extension of such result for other loading conditions determines a transformation criterion useful for macroscopic modelling. This point is on first importance for engineering applications.

In this paper only isothermal loading is discussed, good results are recently obtained in the same way for anisothermal loading path [21]. Improvements remain to bring into the description of hysteresis sub-loop trajectories and it is necessary to introduce the influence of defects on the transformation to describe cyclic behavior and two way-shape memory effect [22].

\section{References}

[1] Otsuka K., Wayman C.M., Nakai K., Sakamoto H. and Shimizu K., Acta metall., 24 (1976) 207226.

[2] Saburi T., Wayman C.M., Tanaka K. and Nenno S., Acta Metall. 28 (1980) 15-32.

[3] Perkins J., Scripta Met. 8 (1974) 1469-1476.

[4] Patoor E., El Amrani M., Eberhardt A. and Berveiller M., Procs. ESOMAT'94, J. Physique IV, 5 (1995) C2-495-500.

[5] Patoor E., Eberhardt A. and Berveiller M., Arch.Mech. 40 (1988) 775-794.

[6] Gillet Y., Patoor E. and Berveiller M., Procs. ESOMAT 94, J. Physique IV, 5 (1995) C2-343-348.

[7] Wechsler M.S., Lieberman D.S. and Read T.A.,Trans AIME 197 (1953) 1503-1515.

[8] Buisson M., Patoor E. and Berveiller M., C.R. Acad. Sci. Paris 313, Série II (1991) 587-590.

[9] Ortin J, and Planes A., Acta Metall. 36 (1988) 1873-1889.

[10] Patoor E., Bensalah M.O., Eberhardt A. and Berveiller M., Procs. ICOMAT'92, Monterey, (CA), USA (1993) 401-406.

[11] Raniecki B., Lexcellent C. and Tanaka K., Arch.Mech. 3 (1992) 261-284.

[12] Sun Q.P. and Hwang K. C., J. Mech. Phys. Solids 41 (1993) 1-33.

[13] Berveiller M. and Zaoui A., J. Engng. Mat. and Technology 106 (1984) 295-299.

[14] Berveiller M., Morreale J. and Reubrez E., Revue Européennes des éléments finis 3 (1994) 491514.

[15] Moumni Z. and Nguyen Q.S., this conference.

[16] Lipinski P. and Berveiller M., Int. J. of Plasticity 5 (1989) 149-172.

[17] Patoor E., Eberhardt A. and Berveiller M., Pitman Research Notes in Mathematics Series 296 (1993) 38-54.

[18] Kröner E., J. Mech. Phys, Solids 25 (1977) 137.

[19] De Vos J., Aernoudt E. and Delaey L., Z. Metallkde 69 (1978) 438-444.

[20] Vacher P. and Lexcellent C, Procs. ICM 6, Kyoto, Japan (1991) 231-236.

[21] Entemeyer D., Patoor E., Eberhardt A. and Berveiller M., Procs. ICOMAT"95, Lausanne, to appear.

[22] Patoor E., Siredey N., Eberhardt A. and Berveiller M., Procs. ICOMAT'95, Lausanne, to appear. 